\title{
Biophysical Modelling of the Triadic Synapse in the Lateral Geniculate Nucleus
}

\author{
Laura Lazzari $^{1,2^{*}}$, Patrick T. McCarthy ${ }^{1 *}$, Jonathan Martin ${ }^{1}$, Simon R. Schultz ${ }^{1}$ \\ ${ }^{1}$ Department of Bioengineering, ${ }^{2}$ School of Medicine, Imperial College London \\ South Kensington, London, SW7 2AZ, United Kingdom \\ laura.lazzari16@imperial.ac.uk; patrick.mccarthy17@imperial.ac.uk \\ jonathan.martin17@imperial.ac.uk; s.schultz@imperial.ac.uk \\ *contributed equally
}

\begin{abstract}
The lateral geniculate nucleus (LGN) is responsible for transmitting visual information from the optic nerve to the primary visual cortex. Located within the LGN is the triadic synapse, an unusual yet fundamental structure wherein a retinal ganglion cell simultaneously synapses onto a relay cell and an interneuron, with the same interneuron also providing inhibition to the relay cell. Despite the large body of physiological data available for each of these cell types individually, the triadic synapse's behaviour and function in information processing remains poorly understood. In this work, we create a biophysical model of the triadic synapse using Python with Neuron. Our model is based on specifications from literature and consists of retinal ganglion inputs, an interneuron and a relay cell synapsing in appropriate triad formation. Computational simulations through the model find that triadic inhibition alone causes faster neuronal repolarisation following excitation than axonal inhibition alone, granting temporal precision to visual signals. Importantly, we find that our triad model expresses temporal selectivity by boosting coincident retinal spikes to selectively pass significant visual events over network noise. This occurs as synchronous retinal inputs elicit a strong relay cell response, whilst asynchronous inputs produce overlapping excitation and inhibition, thus driving relay cells less effectively. This validates the feasibility of temporal selectivity as a core functional property of the synapse and compounds current computational research in investigating triadic circuitry behaviour.
\end{abstract}

Keywords: Interneurons, Action Potentials, Triadic Synapses, Lateral Geniculate Nucleus, Visual Processing

\section{Introduction}

In the mammalian visual system, the lateral geniculate nucleus (LGN) is the thalamic structure responsible for transmission of visual information from the optic nerve to the primary visual cortex (V1) [1]. Its main constituent cells are relay cells (RCs), which project to the visual cortex, and interneurons (INs). Although the LGN was traditionally viewed as a passive relay station, recent literature suggests it may modulate visual signals before they reach V1 and therefore play an important role in processing visual information [2], [3]. As its precise purpose is still not fully understood, computational modelling is essential to develop a mechanistic understanding of circuitry function.

The triadic synapse, formed between RCs, INs and retinal ganglion cells (RGCs) within LGN circuitry, holds key importance in this modulatory process. Within this arrangement, an IN dendritic terminal simultaneously receives excitatory input from a RGC and provides dendrodendritic inhibition to a RC, with the RGC also providing direct excitation to the RC [4], [5]. The same IN provides direct axosomatic inhibition to the RC [6]. In comparison to other neuronal systems, the triadic synapse remains remarkably unexplored, perhaps due to its unusual structural complexity.

Despite the triad's prominent position within the early visual pathway, limited attempts have been made to elucidate its significance. An emerging theory for its function is temporal selectivity, by which triads may increase temporal precision of $\mathrm{RC}$ responses [7]. It is postulated that synchronous retinal inputs may have a summative excitatory effect on the RC preceding IN inhibition [8], while asynchronous spikes produce overlapping excitation and inhibition within the synapse and therefore permit only poor transmission of action potentials. As a result, synchronous RGC inputs are much more effective at driving an RC response than asynchronous inputs [7], [9]. Triadic circuits would therefore preferentially boost coincident retinal spikes to selectively pass significant visual events over network noise [10]. However, there is a notable lack of research investigating this hypothesis. 
Computational modelling is essential in understanding the function of the triadic synapse within the LGN. Neuronal models that simulate RCs or INs independently are scarce [5], [11], [12]. Moreover, to our knowledge, only a single published study to date integrates both RCs and INs in a single model simulate the triadic synapse [13]. While this work provides the foundations for further investigation into triadic activity, it is highly complex and requires high computing power to run. Consequently, through this work we aimed to create an accessible, simple model.

\section{Materials and Methods \\ 2.1 Software}

Modelling was carried out using NEURON, a widely used simulation environment for building both single-neuron and complex neural network models de novo. The bulk of the code was written in Python 3, with NEURON essentially functioning as library. LFPy, a Python package that simulates local field potentials and functions as an API to enable increased model complexity, was used to integrate additional membrane mechanisms from [13] into our IN model to simulate an extensive range of ion channels.

\subsection{Modelling}

An initial model was created by building and connecting single IN and RC cells with excitatory inputs from a single RGC added using the 'NetStim'/Exp2Syn' stimulus protocol outlined below. The IN model was built based on specifications from [13], with additional complexity from [12]. It consists of a cell body (diameter $17.4 \mu \mathrm{m}$, length $15.3 \mu \mathrm{m}$ ) connected to a single dendrite and a single axon with the same dimensions (proximal diameter $4 \mu \mathrm{m}$ tapering to $0.3 \mu \mathrm{m}$ over length $100 \mu \mathrm{m}$, then consistent diameter $0.3 \mu \mathrm{m}$ for distal $400 \mu \mathrm{m}$ ). The RC was assumed to be electrotonically compact [14], and thus took the form of a single-compartment soma (diameter $47 \mu \mathrm{m}$, length $50 \mu \mathrm{m}$ ) [15]. Biophysical parameters were adjusted such that the model exhibited biologically accurate behaviour in response to basic stimuli. Synaptic weights were chosen based on literature to support fast dendrodendritic time-locked inhibition and lagged axosomatic inhibition [16]. The primary purpose of this model was to evaluate the mechanism chosen for modelling the triadic synapse and test the resulting inhibition properties of the model before extending the model to incorporate multiple inputs.

Following single-input model parameterisation, experimental confirmation was obtained that the model expressed appropriate triadic behaviour. Further complexity was added by increasing the number of RGCs that synapse onto the RC, from one to three, concurrent with the number of RGCs found to synapse onto a single LGN RC in current literature [17]. To investigate temporal selectivity, alterations to the stimulus protocol were made as outlined below. Temporal selectivity was tested by specifying that each of the simulated RGC inputs be delivered at a different time and observing the efficacy of each input for eliciting an action potential in the $\mathrm{RC}$ in response. Inhibition mechanisms were then isolated to investigate their contributions to the observed $\mathrm{RC}$ response.

\subsection{Stimulus Protocol}

In the initial model, NEURON objects 'NetStim', 'NetCon' and 'Exp2Syn' were used to generate spike trains which depolarised the cell membrane to a specified potential. When simulating multiple RGC inputs applied at different times, this approach was no longer sufficient to yield accurate results as it did not produce the desired summative behaviour of action potentials. Therefore, in the multi-input model, the NEURON object 'IClamp' was used to simulate the effect of the input stimuli as excitatory postsynaptic current (EPSC) injections. With this approach, the EPSCs exhibit temporal summative behaviour, therefore simulating the behaviour of excitatory postsynaptic potentials (EPSPs) observed in real biological systems [18]. At this stage, we also improved biological accuracy by incorporating additional membrane mechanisms, including but not limited to L-type and T-type $\mathrm{Ca}^{2+}$-currents, slow $\mathrm{Ca}^{2+}$-dependent $\mathrm{K}^{+}$-currents and $\mathrm{Ca}^{2+}$ vesicle pools. These were also used in the model by Heiberg et al. based on experiments in [19]. 


\section{Results}

Triadic behaviour was initially assessed through a single RGC input model. By isolating component inhibition mechanisms, it was found that triadic inhibition alone generates faster RC repolarisation than axonal inhibition alone. Combined, they exhibit a strong summative repolarisation response (Figure 1A).

The multi-input model allowed for subsequent investigation into triadic synapse dynamics. To test for temporal selectivity, the comparison of RC responses to synchronous versus asynchronous stimuli is shown in Figure 1B. It shows that synchronous inputs produce strong RC depolarisation, with efficacy for eliciting a response decreasing as the delay between inputs increases. Similarly, three synchronous RGC stimuli are more effective at inducing RC response than two synchronous RGCs. The latter are in their turn more effective than a single RGC stimulus (Figure 1E). Increasing the number of synchronous inputs into the triad thereby strengthens output $\mathrm{RC}$ response.

Finally, by modelling the triad with three synchronous RGC inputs we found that speed and intensity of RC depolarisation is similar regardless of inhibition state, while repolarisation occurs faster when triadic inhibition is present (Figure 1C). When the synapse is modelled with three asynchronous inputs, however, we find that RC response with triadic inhibition is weaker than without inhibition, and repolarisation again occurs faster (Figure 1D). Overall, this shows that, with triadic inhibition, synchronous inputs are more effective at driving RC response, while without triad mechanics $\mathrm{RC}$ response remains similar regardless of input synchronicity.
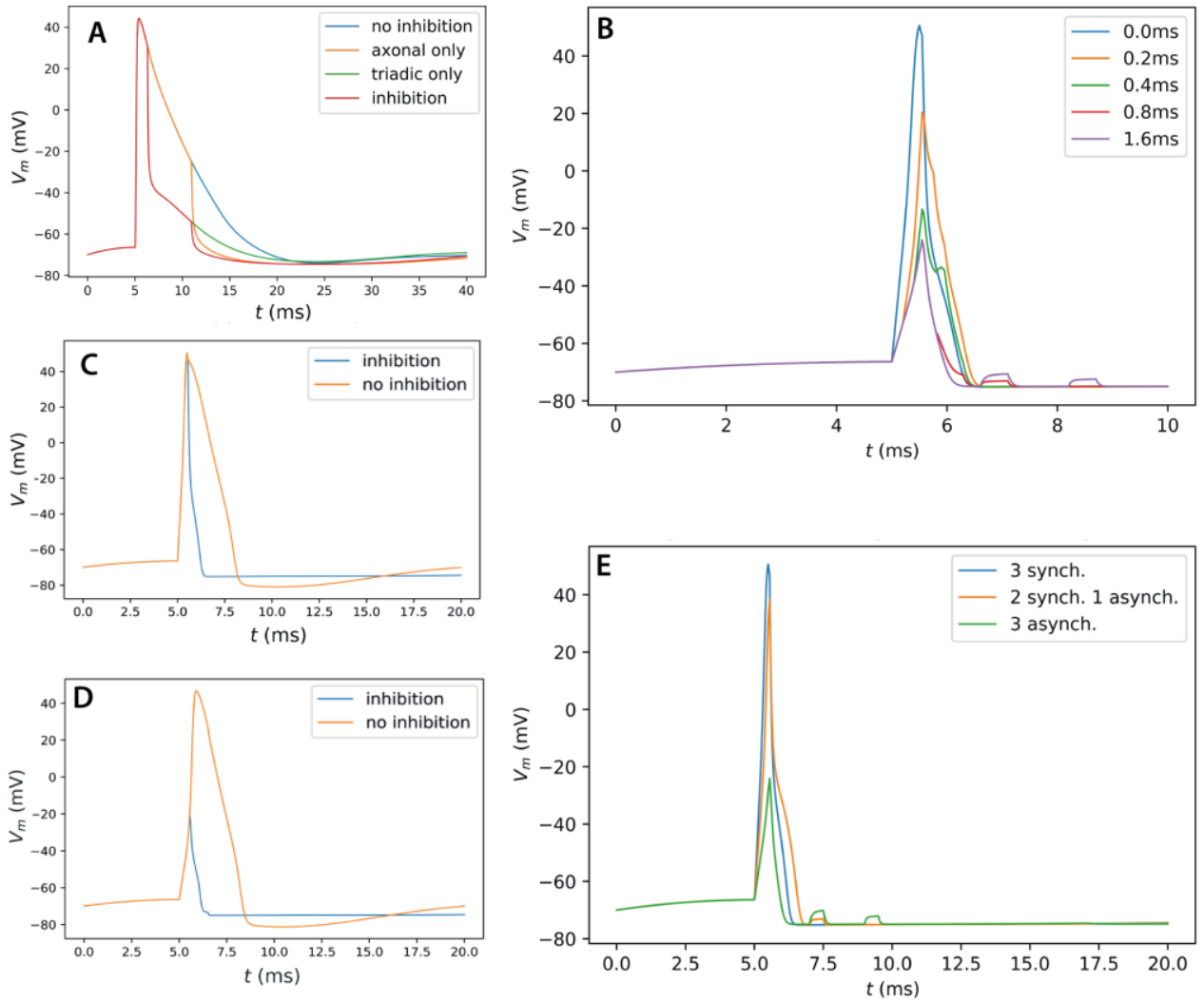

Fig.1: Computational experiments demonstrate that temporal selectivity emerges from triadic inhibition. (A) RC response with isolated inhibition mechanisms. (B) RC response for $3 \mathrm{RGC}$ inputs fired with different delay times. The legend shows interstimulus delay. (C, D) RC response to a range of time-delayed spikes with and without inhibition. (C) 3 simultaneous RGC inputs. (D) 3 RGC inputs mutually delayed by $0.5 \mathrm{~ms}$. (E) RC response for synchronous and asynchronous RGC inputs. The inserted delay for asynchronous inputs is $2 \mathrm{~ms}$. 


\section{Discussion}

Our computational analysis of LGN triadic circuitry has established three salient properties of triadic synapse behaviour. Firstly, we found that triadic inhibition alone activates faster neuronal repolarisation than axonal inhibition. Secondly, we found that synchronous stimuli are more effective at driving RC response with triadic synapses present. Finally, we found that when triadic inhibition is removed, synchronous inputs and asynchronous inputs are equally effective in driving $\mathrm{RC}$ response.

The model's isolated triadic inhibition response (Figure 1A), which allows for faster repolarisation to occur than under standard axonal inhibition, concurs with current literature findings that suggest that the triad could proffer increased temporal precision to visual signals by maintaining a narrow neuronal response [7], [16]. When considering a RC network, this increased temporal precision [20] as a result of synaptic triads may facilitate tightly correlated neuronal spiking within the LGN [21], thereby reinforcing thalamic transmission to the cortex and increasing LGN efficiency in driving cortical targets [22].

The increase in RC response that is observed under synchronous RGC input conditions, compared to a significantly lower response under asynchronous inputs (Figures 1B, 1E), demonstrates that the range of response magnitudes is continuous, resulting from the summation property of excitatory post-synaptic potentials and importantly promoting the likely function of temporal selective filtering within triadic circuitry. This allows significant visual inputs to selectively pass significant visual events over network noise [10].

Finally, by showing that complete removal of triadic inhibition from the model results in similar output products with both synchronous and asynchronous stimuli (Figures 1C, 1D), our model confirms the causative relationship between triadic synapses and temporal selectivity that has been previously only hypothesised in literature.

Our model effectively isolates triadic circuitry and enables an accurate analysis within circuitry function alone. However, by virtue of being a standalone structure, it neglects known complex inputs and outputs into the system. There is potential for future studies to embed the triad within more detailed LGN biophysics to approximate physiological conditions of the entire system and fitting the model with experimental data to finalise accurate parametrisation for specific mammals. Overall, in exclusively studying triadic functionality, our research successfully established a functional and adaptable triadic synapse model that confirms the validity of temporal selectivity as a functional property of the triadic synapse.

\section{Conclusion}

In this study we created a biophysically accurate model of the triadic synapse within the LGN and subsequently confirmed the validity of temporal selectivity as a possible function. Our computational analysis established three salient properties for the behaviour of triadic synapses, namely: triadic inhibition facilitates faster repolarisation than axonal inhibition; synchronous stimuli are more effective at driving relay cells than asynchronous stimuli with triadic synapses present; when triadic synapses are removed, synchronous and asynchronous are equally effective at driving relay cells, thereby confirming the causative relationship between the triad and temporal selectivity.

\section{Code}

The code written for modelling and simulation can be found at: https://github.com/jmckmartin/triadicLGN2020

\section{Acknowledgements}

We would like to thank Prof. Stephen Brickley, Dr. Florian Zirpel and Dr. Gerald Moore from the Department of Life Sciences at Imperial College London for guidance and technical support. We would also like to extend our gratitude to Prof. Gaute Einevoll, Dr. Thomas Heiberg and Dr. Geir Halnes of the Department of Mathematical Sciences and Technology, Norwegian University of Life Sciences for valuable advice. 


\section{References}

[1] J. Kremers, J. H. Kaas, P. R. Martin, and S. G. Solomon, "The Lateral Geniculate Nucleus," Primate Vis. Syst. A Comp. Approach, pp. 161-189, 2006, doi: 10.1002/0470868112.ch6.

[2] Y. B. Saalmann and S. Kastner, "Cognitive and Perceptual Functions of the Visual Thalamus," Neuron, vol. 71, no. 2, pp. 209-223, 2011, doi: 10.1016/j.neuron.2011.06.027.

[3] J. Tang, S. C. A. Jimenez, S. Chakraborty, and S. R. Schultz, "Visual receptive field properties of neurons in the mouse lateral geniculate nucleus," PLoS One, vol. 11, no. 1, pp. 1-34, 2016, doi: 10.1371/journal.pone.0146017.

[4] D. N. Mastronarde, "Nonlagged relay cells and interneurons in the cat lateral geniculate nucleus: Receptive-field properties and retinal inputs," Vis. Neurosci., vol. 8, no. 5, pp. 407-441, 1992, doi: 10.1017/S0952523800004934.

[5] S. M. Sherman, "Interneurons and triadic circuitry of the thalamus," TRENDS Neurosci., vol. 27, no. 11, 2004, doi: 10.1016/j.tins.2004.08.003.

[6] A. M. Sillito, H. E. Jones, G. L. Gerstein, and D. C. West, "Feature-linked synchronization of thalamic relay cell firing induced by feedback from the visual cortex," Nature, vol. 369, no. 6480, pp. 479-482, Jun. 1994, doi: 10.1038/369479a0.

[7] J. L. Morgan and J. W. Lichtman, "An individual interneuron participates in many kinds of inhibition and spans much of the mouse visual thalamus," bioRxiv, p. 683276, 2019, doi: 10.1101/683276.

[8] M. Carandini, J. C. Horton, and L. C. Sincich, "Thalamic filtering of retinal spike trains by postsynaptic summation," J. Vis., vol. 7, no. 14, pp. 20-20, Dec. 2007, doi: 10.1167/7.14.20.

[9] X. Wang, F. T. Sommer, and J. A. Hirsch, "Inhibitory circuits for visual processing in thalamus," Curr. Opin. Neurobiol., vol. 21, no. 5, pp. 726-733, 2011, doi: 10.1016/j.conb.2011.06.004.

[10] W. M. Usrey and R. C. Reid, "SYNCHRONOUS ACTIVITY IN THE VISUAL SYSTEM," Annu. Rev. Physiol., vol. 61, no. 1, pp. 435-456, Mar. 1999, doi: 10.1146/annurev.physiol.61.1.435.

[11] P. A. Rhodes and R. Llinás, "A model of thalamocortical relay cells," J. Physiol., vol. 565, no. 3, pp. 765-781, 2005, doi: 10.1113/jphysiol.2004.070888.

[12] G. Halnes, S. Augustinaite, P. Heggelund, G. T. Einevoll, and M. Migliore, "A multi-compartment model for interneurons in the dorsal lateral geniculate nucleus," PLoS Comput. Biol., vol. 7, no. 9, 2011, doi: 10.1371/journal.pcbi.1002160.

[13] T. Heiberg, E. Hagen, G. Halnes, and G. T. Einevoll, "Biophysical Network Modelling of the dLGN Circuit: Different Effects of Triadic and Axonal Inhibition on Visual Responses of Relay Cells," PLoS Comput. Biol., vol. 12, no. 5, 2016, doi: 10.1371/journal.pcbi.1004929.

[14] S. A. Bloomfield and S. M. Sherman, "Dendritic current flow in relay cells and interneurons of the cat's lateral geniculate nucleus.," Proc. Natl. Acad. Sci. U. S. A., vol. 86, no. 10, pp. 3911-3914, May 1989, doi: 10.1073/pnas.86.10.3911.

[15] A. Destexhe, T. Bal, D. A. Mccormick, and T. J. Sejnowski, "Ionic mechanisms underlying synchronized oscillations and propagating waves in a model of ferret thalamic slices," J. Neurophysiol., vol. 76, no. 3, pp. 20492070, 1996, doi: 10.1152/jn.1996.76.3.2049.

[16] L. E. Vigeland, D. Contreras, and L. A. Palmer, "Synaptic mechanisms of temporal diversity in the lateral geniculate nucleus of the thalamus," J. Neurosci., vol. 33, no. 5, pp. 1887-1896, 2013, doi: 10.1523/JNEUROSCI.4046-12.2013.

[17] Y. K. Hong and C. Chen, "Wiring and rewiring of the retinogeniculate synapse," Current Opinion in Neurobiology, vol. 21, no. 2. NIH Public Access, pp. 228-237, Apr-2011, doi: 10.1016/j.conb.2011.02.007.

[18] J. H. Byrne, Postsynaptic Potentials and Synaptic Integration, Third Edit. Elsevier Inc., 2014.

[19] J. J. Zhu, D. J. Uhlrich, and W. W. Lytton, "Burst firing in identified rat geniculate interneurons," Neuroscience, vol. 91, no. 4, pp. 1445-1460, 1999, doi: 10.1016/S0306-4522(98)00665-4.

[20] D. M. Blitz and W. G. Regehr, "Timing and specificity of feed-forward inhibition within the LGN," Neuron, vol. 45, no. 6, pp. 917-928, 2005, doi: 10.1016/j.neuron.2005.01.033. 
[21] Y. Dan, J. M. Alonso, W. M. Usrey, and R. C. Reid, "Coding of visual information by precisely correlated spikes in the lateral geniculate nucleus," Nat. Neurosci., vol. 1, no. 6, pp. 501-507, 1998, doi: 10.1038/2217.

[22] M. Ghodrati, S. M. Khaligh-Razavi, and S. R. Lehky, "Towards building a more complex view of the lateral geniculate nucleus: Recent advances in understanding its role," Prog. Neurobiol., vol. 156, pp. 214-255, 2017, doi: 10.1016/j.pneurobio.2017.06.002. 